\title{
Duodenal bleeding caused by pancreatic tuber- culosis in a patient with AIDS and disseminated tuberculosis
}

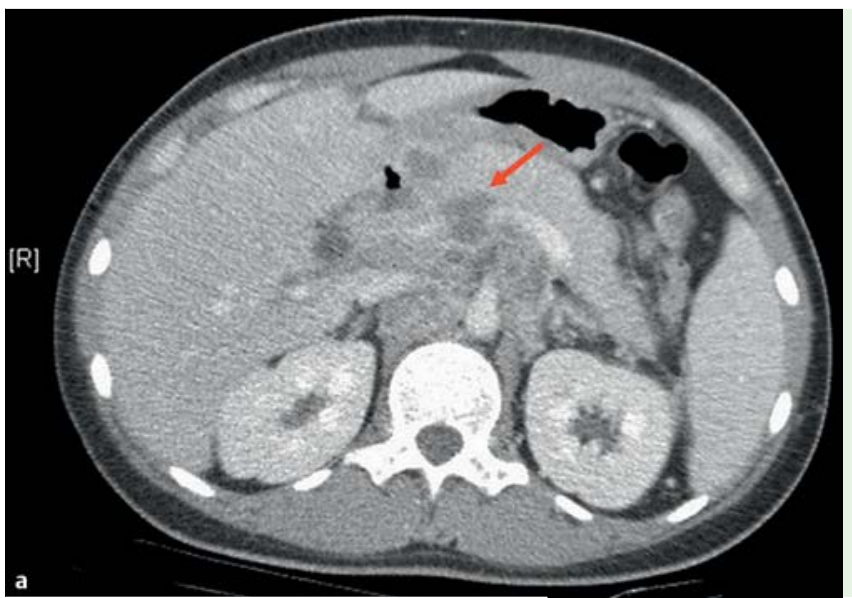

Fig. 1 Abdominal computed tomography (CT) scan showing multiple small low-attenuated masses in the pancreas (arrow) with involvement and compression of the adjacent duodenum: $\mathbf{a}$ in axial view; $\mathbf{b}$ in coronal view.
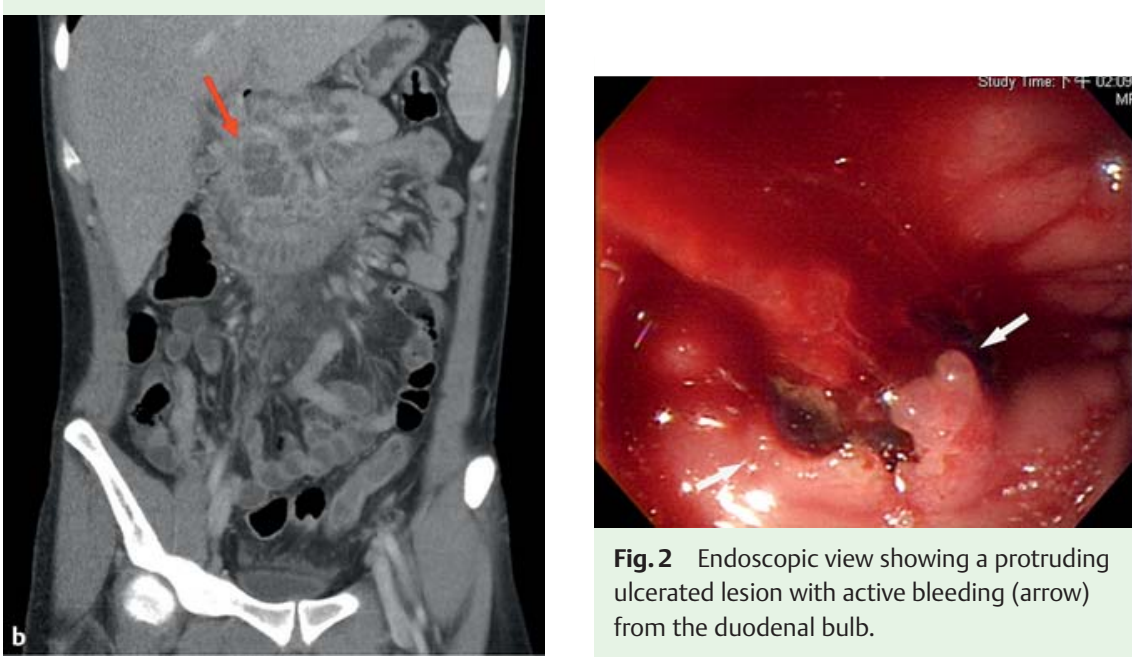

Fig. 2 Endoscopic view showing a protruding ulcerated lesion with active bleeding (arrow) from the duodenal bulb.

Pancreatic tuberculosis is a rare but increasingly recognized disease [1]. Its common clinical manifestations include pancreatic masses that can mimic carcinoma, pancreatitis, and obstructive jaundice [2]. We report a unique case of pancreatic tuberculosis presenting with acute upper gastrointestinal bleeding due to duodenal involvement.

A 22-year-old homosexual man presented with fever, periumbilical pain, and postprandial vomiting of 1 month's duration. Abdominal computed tomography (CT) scans showed multiple low-attenuation pancreatic masses with duodenal involvement, and peripancreatic multiple lymphadenopathy ( Fig.1). Infection with human immunodeficiency virus (HIV) was confirmed by Western blot assay. The patient's CD4+T-cell count was from the duodenal bulb ( $\bullet$ Fig. 2). fast bacilli ( Fig. 5). Polymerase chain reaction assays for Mycobacterium tuberculosis performed on tissue from the pancreatic mass, sputum, ascites, and pleural fluid were all positive.

To our knowledge, there has been only one case report of upper gastrointestinal bleeding caused by pancreatic tuberculosis, in which the patient died from the bleeding and was diagnosed postmortem [3]. The present case confirms that pancreatic tuberculosis can erode the duodenum causing massive upper gastrointestinal bleeding. Treatment of the hematemesis by both endoscopic and surgical methods may be difficult because of brisk bleeding and necrosis of the pancreaticoduodenal tissue. TAE has been reported as an alternative salvage technique to surgery in patients with bleeding peptic ulcers in whom endoscopic hemostasis has not been achieved [4]. This case highlights that TAE is also effective for controlling the duodenal bleeding caused by pancreatic tuberculosis.

Endoscopy_UCTN_Code_CCL_1AB_2AZ_3AD

\section{Competing interests: None}

\section{C.-W. Cheng' ${ }^{1}$, Y.-K. Tsou' ${ }^{2}$, G.-W. Fan², K. P.-H. Wu ${ }^{3}$, C.-C. Yang ${ }^{1}$, W.-Y. Chuang ${ }^{4}$, M.-H. Lee ${ }^{1}$}

11 cells/ $\mu \mathrm{L}$ and his plasma HIV RNA viral load was 1271795 copies/mL. During his first week in hospital he experienced massive hematemesis, and urgent esophagogastroduodenoscopy revealed a protruding, ulcerated lesion with active bleeding

Endoscopic methods failed to stop the hematemesis and he therefore proceeded to surgical treatment. An indurated pancreatic mass invading into the posterior wall of the duodenal bulb was confirmed, but surgical treatment for the hemostasis was difficult. Angiography showed extravasation of contrast medium from the right hepatic artery ( $\bullet$ Fig. 3 ) and transarterial embolization (TAE) was successfully used to control the bleeding ( Fig.4). Microscopically, the pancreatic mass was composed of small granulomas and acid-
${ }^{1}$ Division of Infectious Diseases, Department of Internal Medicine, Chang Gung Memorial Hospital and Chang Gung University College of Medicine, Taoyuan, Taiwan

2 Division of Gastroenterology and Hepatology, Department of Internal Medicine, Chang Gung Memorial Hospital and Chang Gung University College of Medicine, Taoyuan, Taiwan

${ }^{3}$ Department of Physical Medicine and Rehabilitation, Chang Gung Memorial Hospital and Chang Gung University College of Medicine, Taoyuan, Taiwan ${ }^{4}$ Department of Pathology, Chang Gung Memorial Hospital and Chang Gung University College of Medicine, Taoyuan, Taiwan 

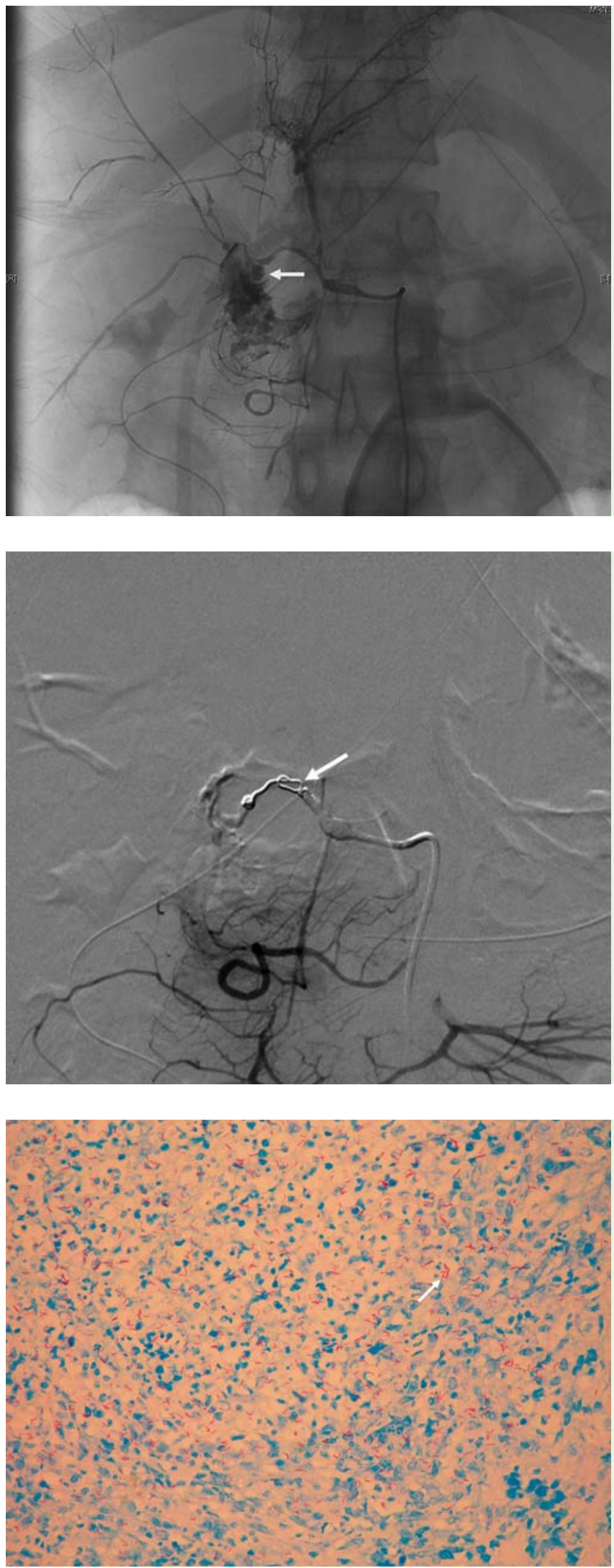

Fig.3 Angiographic image showing extravasation of contrast medium from the right hepatic artery (arrow).

\section{References}

1 Nagar AM, Raut AA, Morani AC et al. Pancreatic tuberculosis: a clinical and imaging review of 32 cases. J Comput Assist Tomogr 2009; 33: $136-141$

2 Franco-Paredes C, Leonard M, Jurado R et al. Tuberculosis of the pancreas: report of two cases and review of the literature. Am J Med Sci 2002; 323: 54-58

3 Fan ST, Yan KW, Lau WY et al. Tuberculosis of the pancreas: A rare cause of massive gastrointestinal bleeding. Br J Surg 1986; 73 : 373

4 Wong TC, Wong KT, Chiu PW et al. A comparison of angiographic embolization with surgery after failed endoscopic hemostasis to bleeding peptic ulcers. Gastrointest Endosc 2011; 73: 900-908

\section{Bibliography}

DOI http://dx.doi.org/

10.1055/s-0031-1291514

Endoscopy 2012; 44: E34-E35

(c) Georg Thieme Verlag KG

Stuttgart · New York

ISSN 0013-726X

image following suc cessful control of bleeding after embolization with two microcoils and gelfoam cubes (arrow).

Fig. 5 Microscopic appearance of the pancreatic mass showing numerous scattered acid-fast bacilli (arrow; Ziehl-Neelsen stain, original magnification $\times 400$ ).

\section{Corresponding author}

\section{M.-H. Lee, MD}

Division of Infectious Diseases Department of Internal Medicine Chang Gung Memorial Hospital

5 Fu-Shin St.

Gueishan County 333

Taoyuan

Taiwan

Fax: +886-33-289410

drharrylee@gmail.com 